\title{
An Early Assessment of JPSS-1/NOAA-20 VIIRS Day-Night Band On- Orbit Calibration and Performance
}

\author{
H. Chen ${ }^{1}$, H. Oudrari ${ }^{1}$, C. Sun ${ }^{2}$, T. Schwarting ${ }^{2}$ and X. Xiong ${ }^{3}$ \\ ${ }^{1}$ Systems \& Applications, Inc., 10210 Greenbelt Rd, Lanham, MD 20706; \\ ${ }^{2}$ Global Science Technology, Inc., 7855 Walker Drive, Greenbelt, MD 20770; \\ ${ }^{3}$ Science Systems and Exploration Directorate, NASA/GSFC, Greenbelt, MD 20771
}

\begin{abstract}
The JPSS-1 (now named NOAA-20) VIIRS instrument has been successfully operating on orbit since November $28^{\text {th }}$, 2017. The Day-Night Band (DNB) is a panchromatic channel covering wavelengths from 0.5 to $0.9 \mu \mathrm{m}$ that is capable of observing the Earth scene in visible/near-Infrared spectral range at spatial resolution of $750 \mathrm{~m}$. The DNB operates at low, mid, or high radiometric gain stages, and it uses an onboard solar diffuser (SD) panel for low gain stage calibration. The SD observations also provide a mean to compute gain ratios between low-to-mid and mid-to-high gain stages. With their large dynamic range and high sensitivity, the DNB detectors can make observations during both daytime and nighttime. This paper provides an early assessment of the DNB on-orbit performance and behavior in the first 90-day post launch test (PLT) period and beyond. The calibration methodology used by the VIIRS Characterization Support Team (VCST) in support of the NASA earth science community will be presented. The trending of OBC dark-offsets, SD gains and gain ratios, and signal-to-noise ratio (SNR) at minimum radiance have been analyzed, especially during key events such as the Nadir and Cryo-cooler doors opening. Furthermore, we performed inter-comparison studies between SNPP and JPSS-1 instruments and evaluated DNB radiometric calibration and characterization, including the SD degradation, detector gains and gain ratios, as well as the calibration comparison between the IDPS LUTs and our VCST delivery results.
\end{abstract}

Keywords: JPSS-1, VIIRS, VCST, DNB, Calibration, Performance

\section{INTRODUCTION}

The Visible Infrared Imaging Radiometer Suite (VIIRS) on-board the first Joint Polar Satellite System (JPSS-1) spacecraft (now called NOAA-20) was launched on November 18, 2017. Twenty-five days after launched into Earth orbit, JPSS-1 sent back its first VIIRS science data on December 13, 2017 when the nadir door was open, as part of a series of instrument activation and checkouts that is taking place before the satellite goes into operational mode. VIIRS is one of the key five instruments onboard JPSS-1 that will improve day-to-day weather forecast and environmental monitoring. Since its nadir door opened, Earth View (EV) data have been collected for all the reflective solar bands (RSB). The thermal emissive bands (TEB) became operational shortly after January $3^{\text {rd }}$, 2018 when the passive cryocooler door was opened. The VIIRS observations are made in 22 spectral bands, including 16 moderate resolution bands, 5 imaging bands, and one panchromatic Day-Night band (DNB), with wavelengths ranging from 0.4 to $12 \mu \mathrm{m}$. The calibrated and geo-located radiances, reflectance and brightness-temperature are called the Sensor Data Records (SDRs), which are used to generate the Environmental Data Records (EDRs). VIIRS was built and operated with a strong heritage from the Moderate Resolution Imaging Spectroradiometer (MODIS) on-board the Aqua and Terra spacecrafts. The experience from MODIS, including its design, spectral band coverage, on-board calibrators (OBCs), and calibration approaches and strategies, has been beneficial in the subsequent operation of the VIIRS instrument. 
VIIRS OBCs include a flat panel v-grooved blackbody (BB), a solar diffuser (SD) and a SD stability monitor (SDSM) that tracks any SD on-orbit degradation [1]. A rotating telescope (RTA) design was chosen rather than a scan-mirror in order to suppress stray light. The RTA is a three-mirror anastigmatic design such that light out of the RTA reflects onto a mirror that rotates at half the angular velocity of the RTA, called the half-angle mirror (HAM). As RTA rotates, it takes a view of three calibration sectors: The space view (SV) points out to deep space just above the mesospheric limb, and occurs just before the start of the earth view (EV) data collection; the BB view occurs after the RTA sweeps across the Earth and is inside the instrument; and the SD view which is illuminated by the sun through a solar attenuation screen during a 2 to 3 minute period each orbit over the south pole. The SV is an aperture providing a view of cold space to allow for a zero radiance input signal on each scan. The Moon can also be observed via the SV at a frequency of approximately once per month.

DNB is a panchromatic visible/near-infrared (Vis/NIR) band designed to detect radiance from the brightest daytime scenes down to very dim nighttime scenes illuminated by the lunar irradiance. Its dynamic range spans over 7 orders of magnitude. It has a wide spectral bandpass, centered at $0.7 \mu \mathrm{m}$, and uses a temperature-controlled charge-coupled device (CCD). The CCD focal plane array (FPA) is sectioned into 4 stages, where each stage essentially functions as a separate imager, though they all reside on the same FPA. There are total 32 aggregation modes over the full DNB swath resulting in an almost constant ground resolution across the EV. However, the JPSS-1 uses only 22 aggregation modes to compensate for DNB non-linearity issue as seen during the pre-launch thermal vacuum testing.

There are four detector arrays at three different radiometric gains used to cover the wide radiometric dynamic range. Each detector array has 672 sub-pixel detectors along-track, which are aggregated onboard to create sixteen near constant $750 \mathrm{~m}$ along-track pixels for each along-scan frame. The high gain stage (HGS) has two redundant arrays, identified as HGA and HGB. Each HGS array has 250 along-scan sub-pixel detectors operating in time delay integration (TDI) for nighttime imagery. The third array is identified as middle gain stage (MGS), which has 3 along-scan sub-pixel detectors operating in TDI. The final low gain stage (LGS) has a single sub-pixel detector in the scan direction.

In NOAA Interface Data Processing Segment (IDPS) calibrations, the DNB is calibrated through a monthly VIIRS recommended operating procedures (VROPs) and the SD observations [1]. Basically, there are two VROPs: VROP705 is for gain ratio determination, and VROP702 is for offset determination. The VROP data were analyzed to estimate the dark offset and cross-stage gain ratios. The drawback of using VROP is the loss of science data as the instrument in maneuver operations. Furthermore, nighttime airglow contamination may also be embedded in VROP data, and could introduce additional uncertainty. Alternatively, the NASA VIIRS characterization support team (VCST) has proposed a DNB calibration approach that directly utilizes calibration view data to continuously track the gain and offset over time [2]. This method determines the dark offset drift using nighttime observed calibrator dark signals, which is free from airglow contamination. It estimates the cross-stage gain ratios based on simultaneously observed SD signals in each solar calibration event. It can continuously update the offset and gain ratio drift as the calibration view data is continuously collected in a repeatable orbital cycle.

This paper is organized as follows. In Section 2, DNB system behavior has been illustrated, including the SDSM early degradation, DNB wavelength coverage and pre-launch relative spectral response (RSR). The relationship of data sequence and aggregation modes in both the JPSS-1/N20 DNB and the S-NPP are presented. Then, in Section 3 calibration performance and trending results are plotted. Dark offsets, LGS gain and gain ratios are all evaluated. For verifications, Section 4 shows our results comparing with the official NOAA IDPS LUTs. The conclusions of the paper are presented in Section 5.

\section{DNB SYSTEM BEHAVIOR}

The VIIRS SD bi-directional reflectance distribution function (BRDF) was characterized pre-launch. The on-orbit changes of the SD BRDF are monitored by the on-board SDSM. In general, the SDSM is operated during each SD calibration to perform alternate views of the sunlight through a fixed transmission screen (Sun view), and of sunlight diffusely reflected from the SD panel (SD view). The SD on-orbit degradation can be calculated using the ratio of brightness along the two viewing paths. The center wavelength of VIIRS reflective solar bands (RSB) covers a range from $0.412 \mu \mathrm{m}$ to $2.250 \mu \mathrm{m}$. Specifically, we have M1: $0.412 \mu \mathrm{m}$; M2: $0.445 \mu \mathrm{m}$; M3: $0.488 \mu \mathrm{m}$; M4: $0.555 \mu \mathrm{m}$; M5 
$0.672 \mu \mathrm{m}$; M6: $0.746 \mu \mathrm{m}$; M7: $0.865 \mu \mathrm{m}$; M8 $1.240 \mu \mathrm{m}$; M9: $1.375 \mu \mathrm{m}$; M10: $1.610 \mu \mathrm{m}$; M11: $2.250 \mu \mathrm{m}$. The SDSM has 8 detectors covering the wavelength form $412 \mathrm{~nm}$ to $936 \mathrm{~nm}$. Figure 1 shows the VIIRS SDSM degradation early mission, including orbits of 500, 1500, 2500, and 3500. Orbit-3500 was acquired on July 23, 2018, about 8 months of operation since nadir door was open. There is about $10 \%$ degradation at wavelength $0.412 \mu \mathrm{m}$. From the early degradation point of view, the largest degradation occurs in the shortest wavelength as expected. The DNB wavelength coverage is illustrated in this figure as well.

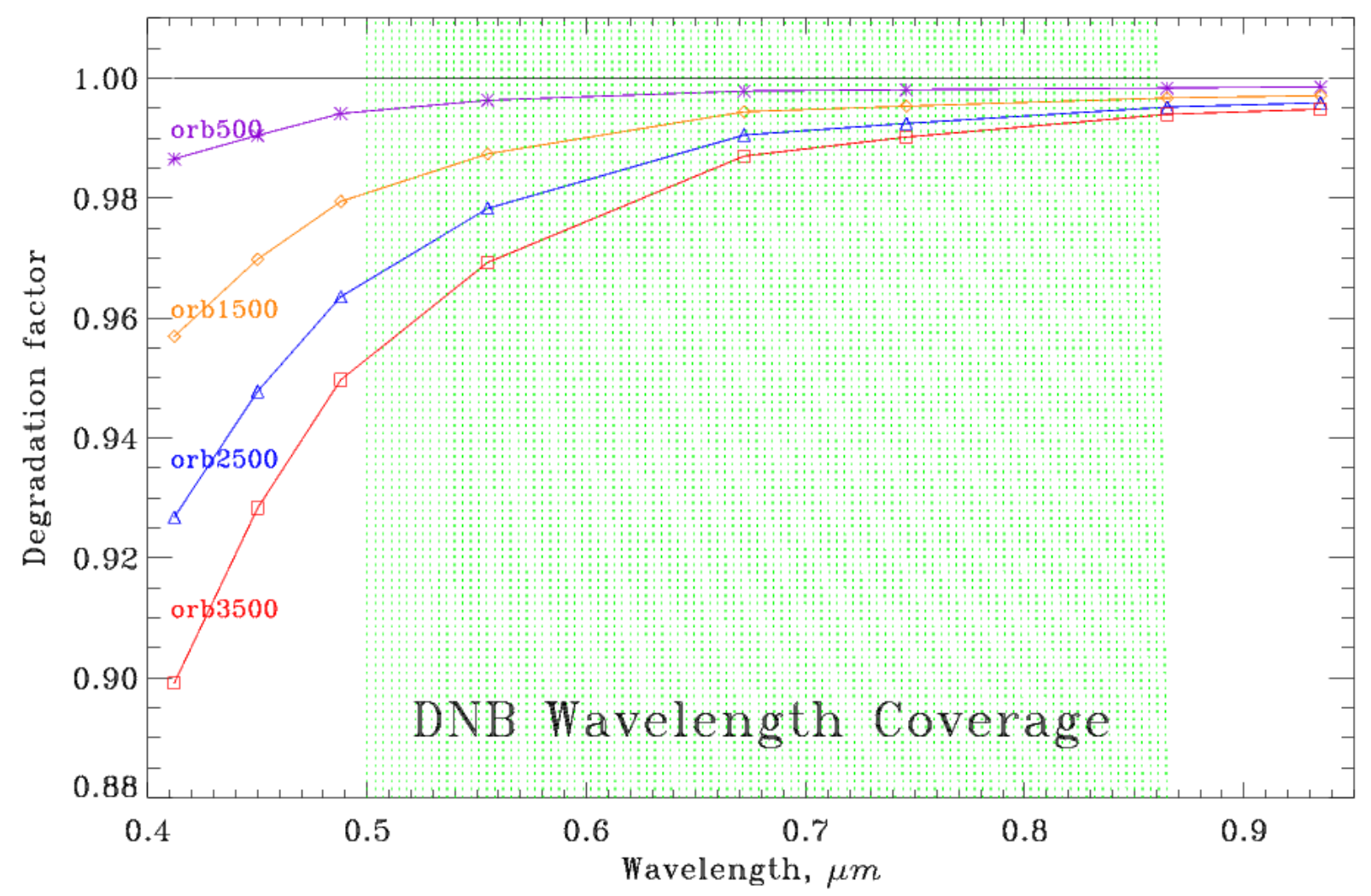

Figure 1 VIIRS SDSM degradation factor for four (4) obits in the early JPSS-1 mission. Curve color represent different orbits, and DNB wavelength coverage is shadowed in the chart.

As the degradation is wavelength dependent, DNB RSR is expected to be significantly affected as it has a wide bandpass wavelength range. Thus, DNB radiometric gain needs to be convolved with its RSR to reflect the change of incident solar radiance. Figure 2 shows the pre-launch measured DNB RSR in JPSS-1 and S-NPP. S-NPP VIIRS RTA mirrors suffer significant degradation since the beginning of the mission due to a pre-launch mirror coating problem [3]. It also causes the DNB RSR to change due to the differences of degradation rate at different wavelengths. The VCST has analyzed and implemented a methodology of modulated-RSR to address this issue. RSR profiles are adjusted in postlaunch processing for all RSB and DNB spectral bands. In practice, the VCST calibration method uses a time-dependent modulated RSR to estimate the DNB LGS gain during solar calibration events for S-NPP. However, in JPSS-1, only prelaunch RSR is used for calibration in the early mission assessment (mirror contamination was eliminated for JPSS-1 VIIRS). Thus, the DNB degradation will be integrated with the corresponding SDSM degradation and the DNB RSR profile. As JPSS-1 VIIRS operation continues, a time dependent RSR will be employed to provide more accurate calibrations in the coming future if needed. Comparing with S-NPP, the JPSS-1 DNB pre-launch measured RSR has much sharper edge response in both lower and higher bandwidth edges, and much better flat responses in the DNB wavelength range. 


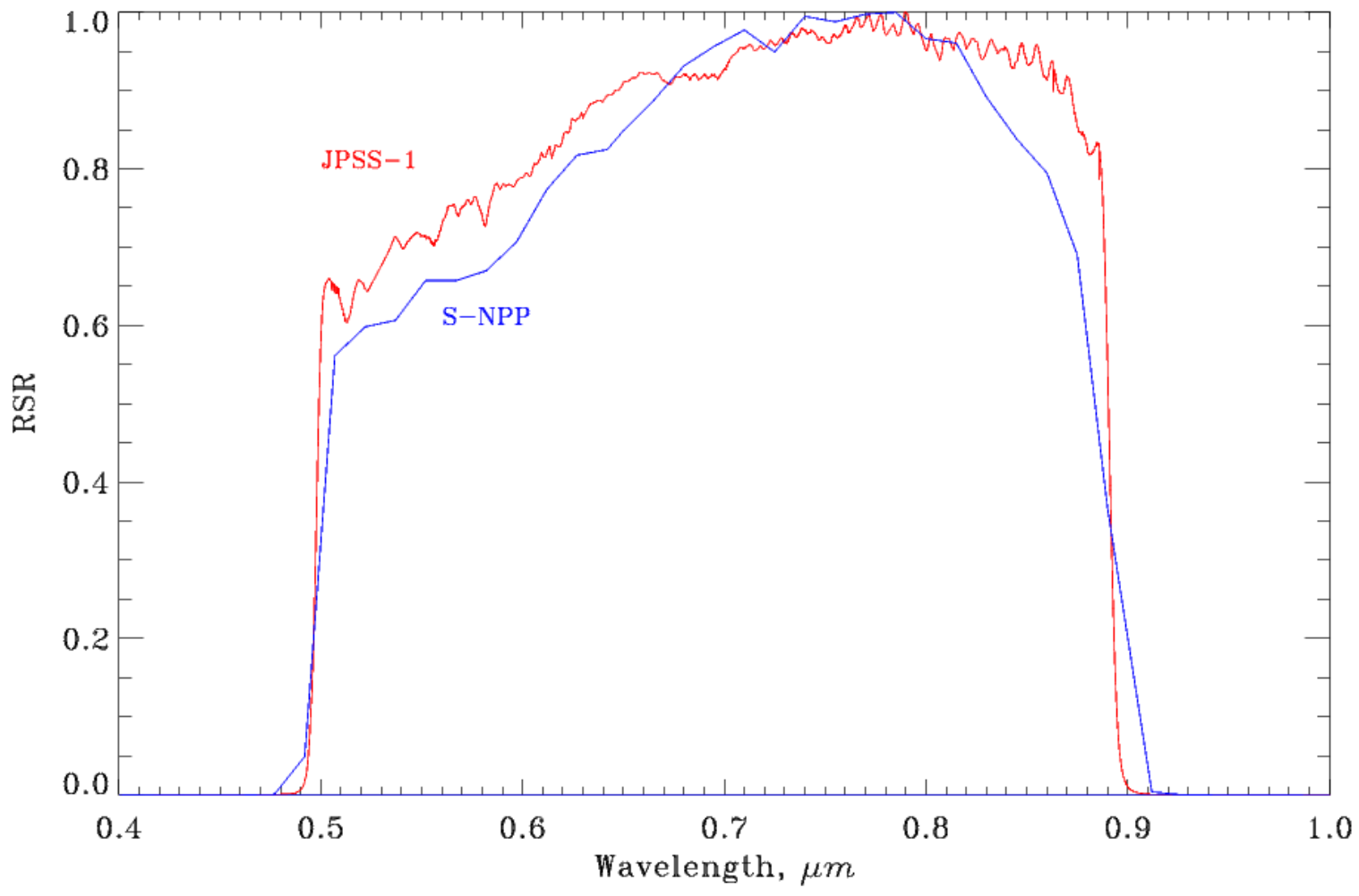

Figure 2 Pre-launch measured DNB RSR JPSS-1 and S-NPP.

To meet the goal of an almost constant ground resolution in all scans, S-NPP has 32 aggregation modes in VIIRS DNB. However, there are only 22 aggregation modes implemented in the JPSS-1 VIIRS DNB. As the hardware platform design is the same for both S-NPP and JPSS-1 DNB sub-systems, the relationship of data sequences and aggradation modes is therefore different so as to provide 22 aggregation modes from 32 possible sequences. Figure 3 plots the relationship maps for both S-NPP and JPSS-1 DNB sub-systems. In the case of S-NPP DNB, sequence and mode are one-by-one related. However, many aggregation modes are duplicated to fit 32 spaces in the JPSS-1 DNB. Note that mode- 1 and mode-32 are with the same data sequences. Both instruments keep a unique relationship of sequence order and mode index from mode- 5 to mode- 17 . 


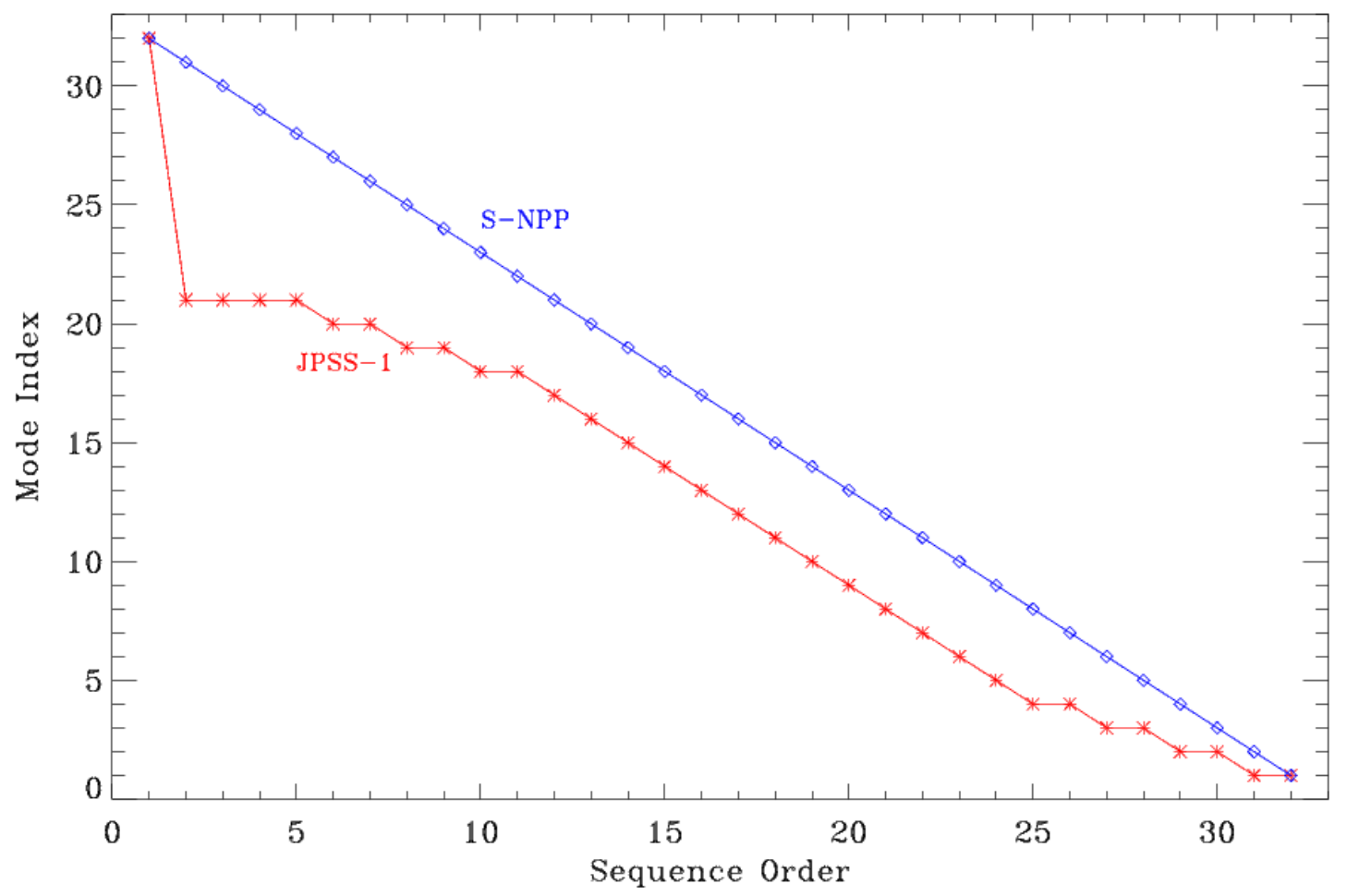

Figure 3 Map of DNB data sequence versus aggregation mode.

\section{CALIBRATION PERFORMANCE AND TRENDING}

The VIIRS DNB is designed to make global observations day and night with a large dynamic range at three different gain stages, LGS, MGS and HGS. Similar to the VIIRS RSB, the primary radiometric calibration coefficient for DNB is the F-factor, which corresponds to the inverse of the DNB gain. It is a linear multiplicative coefficient correcting the prelaunch radiometric coefficients. The principle method for determining the value of the F-factor is the use of the SD data while it is fully illuminated by the Sun over the South Pole once per orbit [1-3]. Specifically, using the SD sweetspot data, defined as the Sun declination angle is between 14 and 18 degrees, the DNB SD radiance is calculated by,

$$
L_{S D}=\frac{\cos \theta_{S D} \cdot R V S_{S D} \cdot \int B R D F(\lambda) \tau_{s d s} H(\lambda, t) R S R(\lambda, t) \phi(\lambda) d ;}{4 \pi d_{S D-S U N}^{2}}
$$

Where $\boldsymbol{\theta}_{S \boldsymbol{D}}$ is the angle between the normal of the SD surface and the SD-to-Sun vector; $\boldsymbol{\Phi}(\lambda)$ is the solar spectral power at a distance of one astronomical unit; $d_{S D-S U N}$ is the Sun-to-spacecraft distance in astronomical units; $\tau_{s d s}$ is the transmittance of the pinhole screen for the SD; $\boldsymbol{H}(\boldsymbol{\lambda}, t)$ denotes the SD degradation index which is both time and wavelength dependent and determined by combining the DNB RSR and the SDSM H-factors, which are degradation estimates come from eight SDSM detectors; $\boldsymbol{B R} \boldsymbol{D F}(\boldsymbol{\lambda})$ is the bidirectional reflectance factor of the SD, and it was characterized prelaunch using reference samples traceable to the National Institute of Standards and Technology reflectance standards; $\boldsymbol{R} \boldsymbol{S R}(\boldsymbol{\lambda}, \boldsymbol{t})$ is the DNB RSR; and $\boldsymbol{R} \boldsymbol{V} \boldsymbol{S}_{\boldsymbol{S O}}$ is the response versus scan angle of the HAM at the SD view angle. The DNB LGS gain coefficients are determined by

$$
L_{S D}=c_{1} \cdot a^{\top} n_{S D}
$$


The background corrected $d \boldsymbol{n}_{s o}$ is computed per detector, aggregation mode, and HAM side for each scan. From the gain coefficients, one can compute the scene radiance using:

$$
L_{E V}=\frac{d n_{E V} \cdot \cos \theta_{S D} \cdot R V S_{S D} \cdot \Delta}{d n_{S D} \cdot R V S_{E V}}
$$

Where

$$
\Delta=\frac{1}{4 \pi a_{S D-S U N}^{2}} \int_{-\infty}^{\infty} B R D F(\lambda) \tau_{s d s} H(\lambda, t) R S R(\lambda) \Phi(\lambda) d \lambda
$$

The JPSS-1/N20 VIIRS DNB calibration is performed periodically to track the on-orbit detector gain change. Due to the high sensitivities of MGS and HGS, the fully illuminated SD signal will saturate detectors in these gain stages and thus cannot be used to compute the gains. However, carefully selected data, such as when both LGS and MGS have appropriate and below saturation values, can be used to derive MGS/LGS ratio. Similarly, HGS/MGS ratio can be derived using the same data criteria as MGS/LGS.

In all, DNB LGS gain calibration is performed for each SD calibration event using the DNB pre-launch RSR at the beginning of JPSS-1 mission. The MGS and HGS gains are computed based on LGS results, together with the estimated gain ratios of HGS/MGS and MGS/LGS. The DNB calibration algorithm is detector, gain-stage, aggregation-mode and HAM-side dependent.

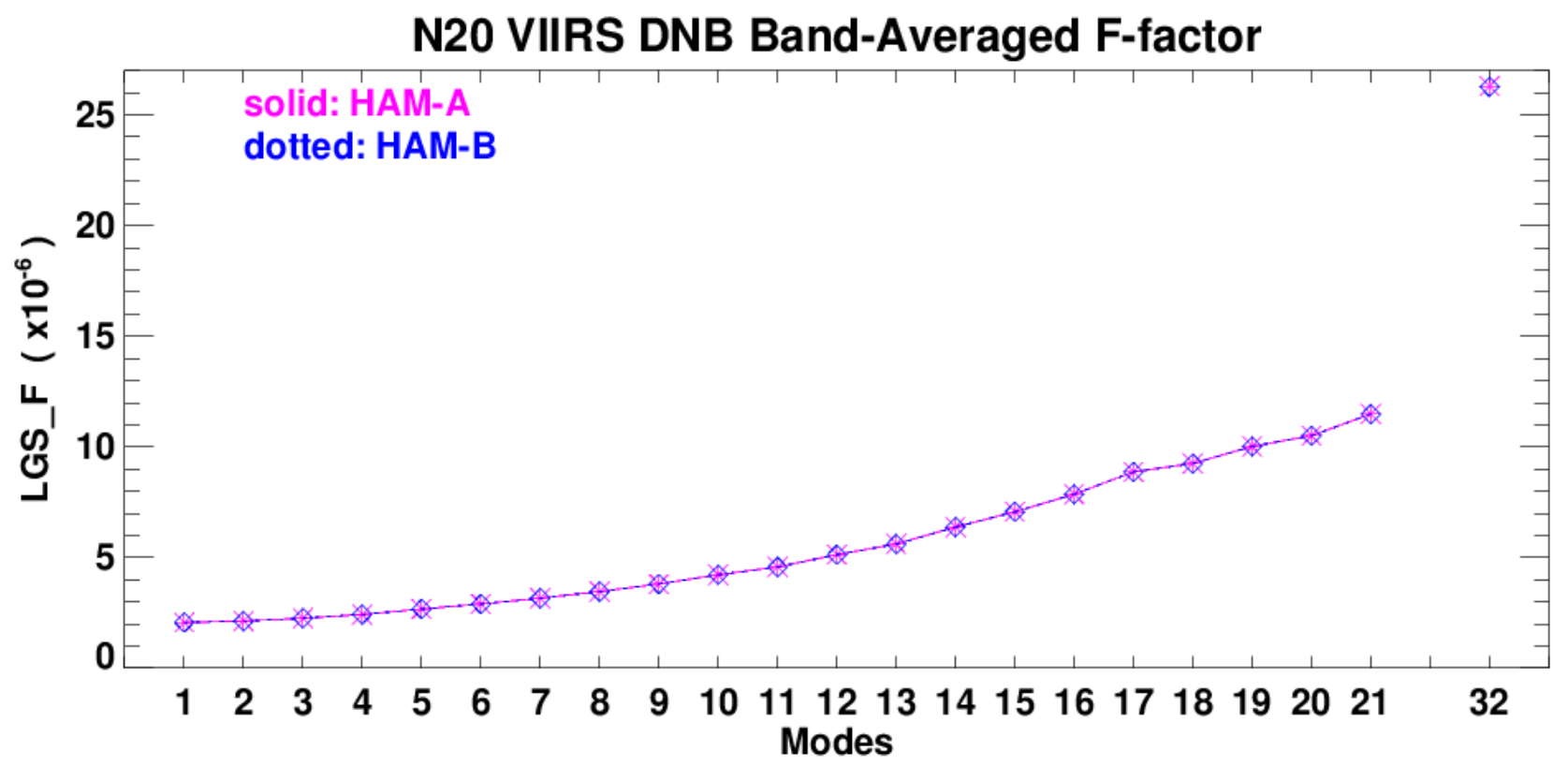

Figure 4 DNB LGS band-averaged F-factor on June 30, 2018.

Figure 4 shows the DNB band-averaged LGS F-factor, inverse of gain units of W. $\mathrm{cm}^{-2} \mathrm{sr}^{-1}$. In this figure, all 22 aggregation modes are included. Since there are not cases of modes 22-31 in the JPSS-1/N20 DNB, the case of mode-21 is just followed be the mode-32, which is not out of family. Both ham sides are with almost identical results. As mode indices increase, the gains decrease as expected, as Mode- 1 is corresponding the NDIR frame, and Mode-32 is the edge frame. 


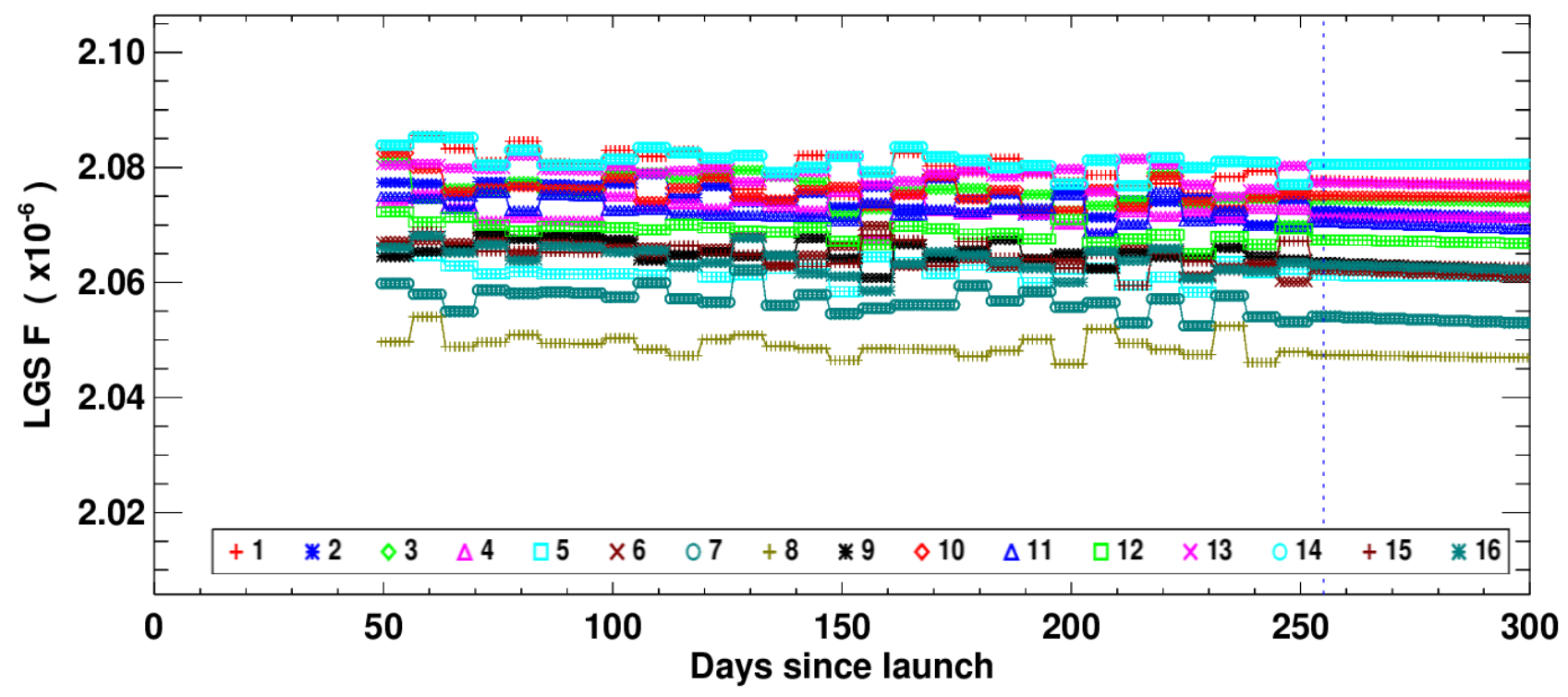

Figure 1 DNB LGS Mode-1 daily averaged LGS gain trending.

Figure 5 plots DNB mode-1officially delivered LGS F-factor trending, which starts from the time of the cryo-door-open. The daily averaged results are selected every 7 days, and the 7-day gups are constantly filled using the previous same value, as we use it whenever the instant time belongs in between. The vertical line denotes the currently measured day. If normalized at the first point, all DNB detectors are observed with similar trending features in this case. In addition, the last 100-days data has been used with a linear fit for forward deliveries. As previously mentioned, the VCST uses the SD signals outside of direct solar illumination to compute the gain ratios between DNB's low-to-mid and mid-to-high gain stage. Figure 6 shows the ratio trend of DNB HGS over MGS using SD signals from aggregation mode-1.

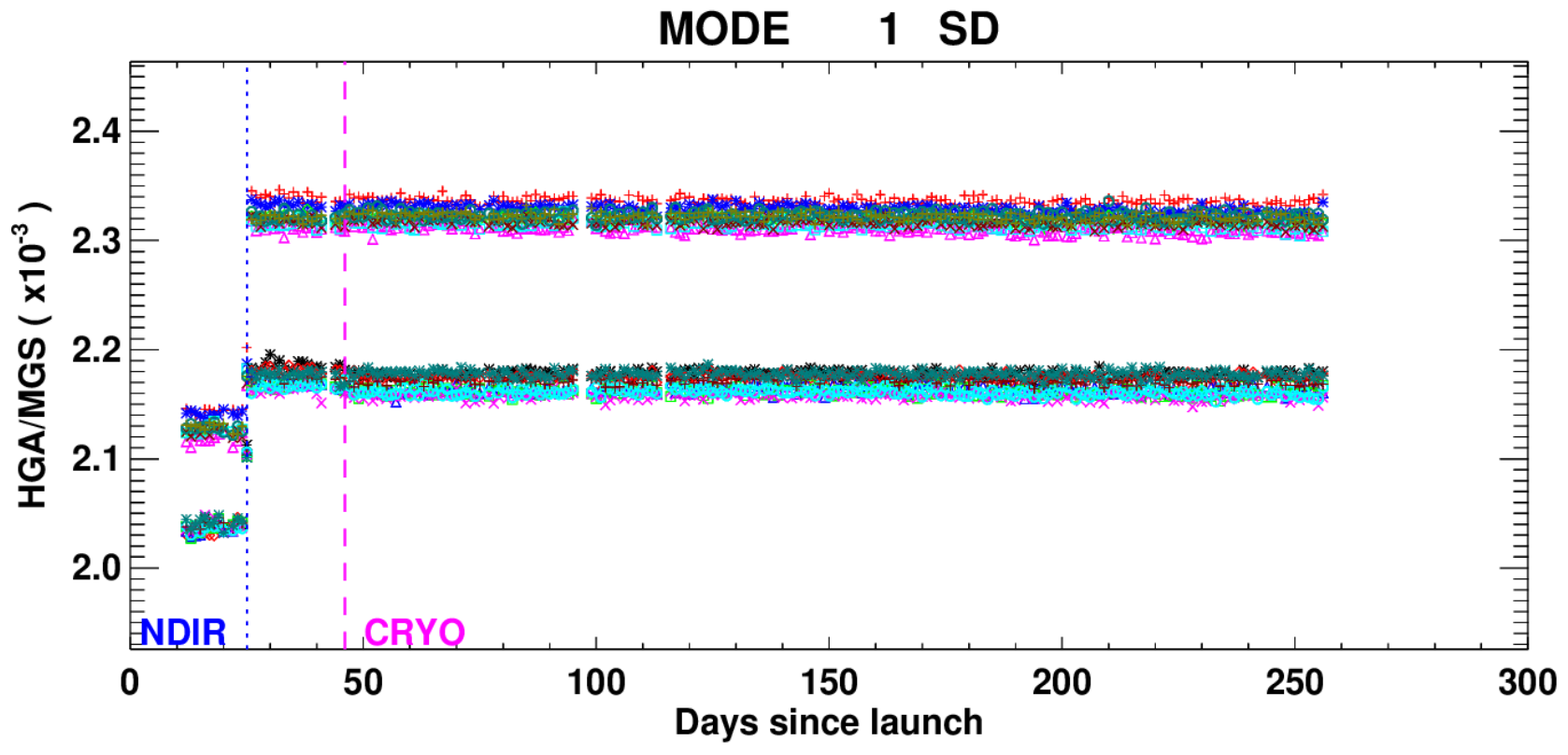

Figure 2 Ratio trend of DNB mode-1, HGS to MGS ratios using SD signals. 
As examples, MGS/HGS ratio trends of DNB mode-32 and mode-21 using SD signals are shown in Figure 7 and Figure 8 , respectively. After assessments for all aggregation modes, it is observed that all ratio results present relatively stable trending for all detectors in the early operations.

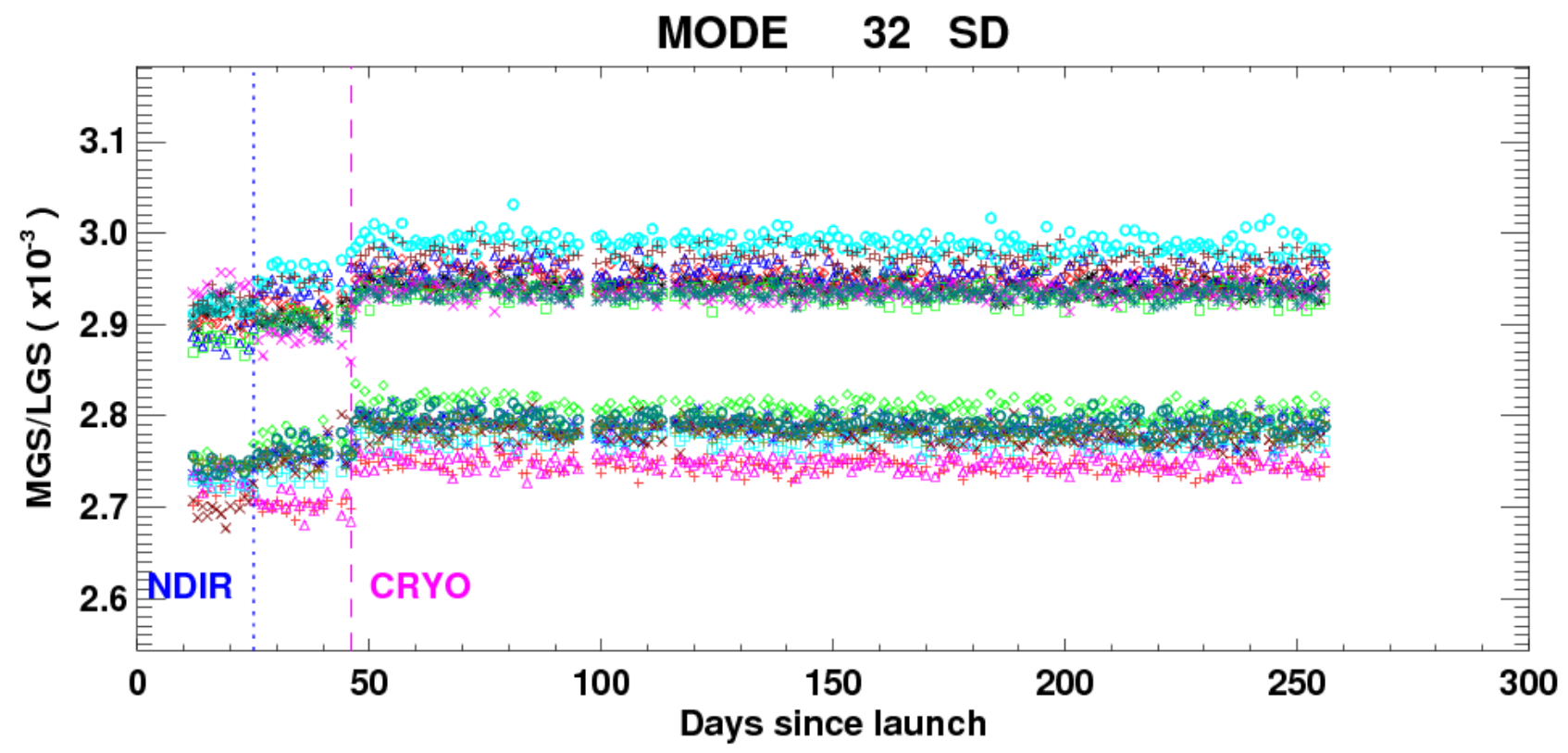

Figure 7 Ratio trend of DNB mode-32 MGS over LGS using SD signals.

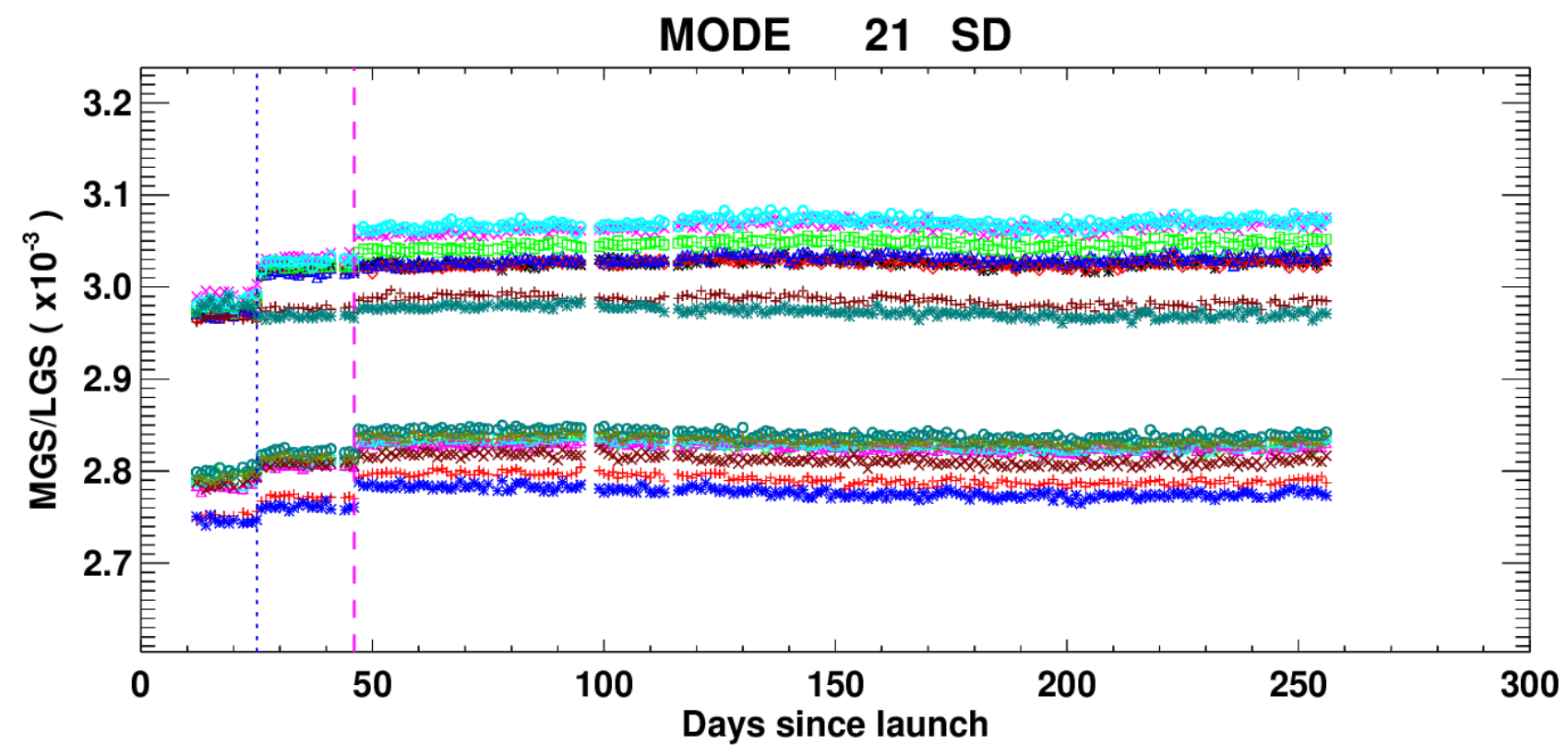

Figure 8 Ratio trend of DNB mode-21 MGS over LGS using SD signals.

Figure 9-11 illustrate DNB dark offsets in three different cases of mode-1, mode-17 and mode-32, respectively. The dark offsets are estimated using all three on-orbit calibrators, SD, BB and SV, among which the minimal dn is selected as the dark offset in each scan. Three event times are plotted, the nadir door opening, the cryo-door opening, and the end of outgassing. Notice that the first two events introduced large drops of dn values, as expected since the instrument 
temperature decreased a lot in these events. However, there is no impact to the dark offset trend during the outgassing event.

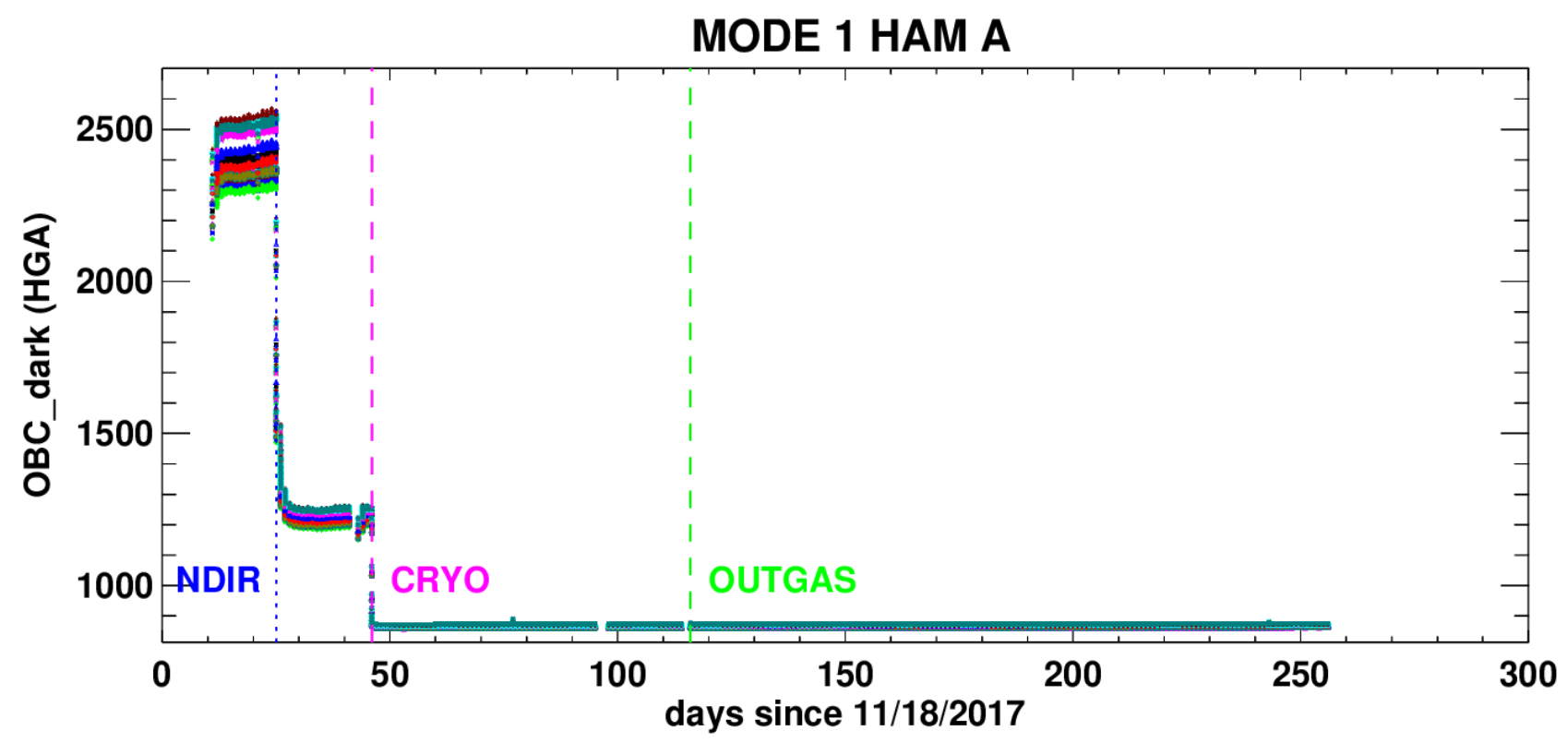

Figure 9 DNB dark offset trending in mode-1 and ham side-A.

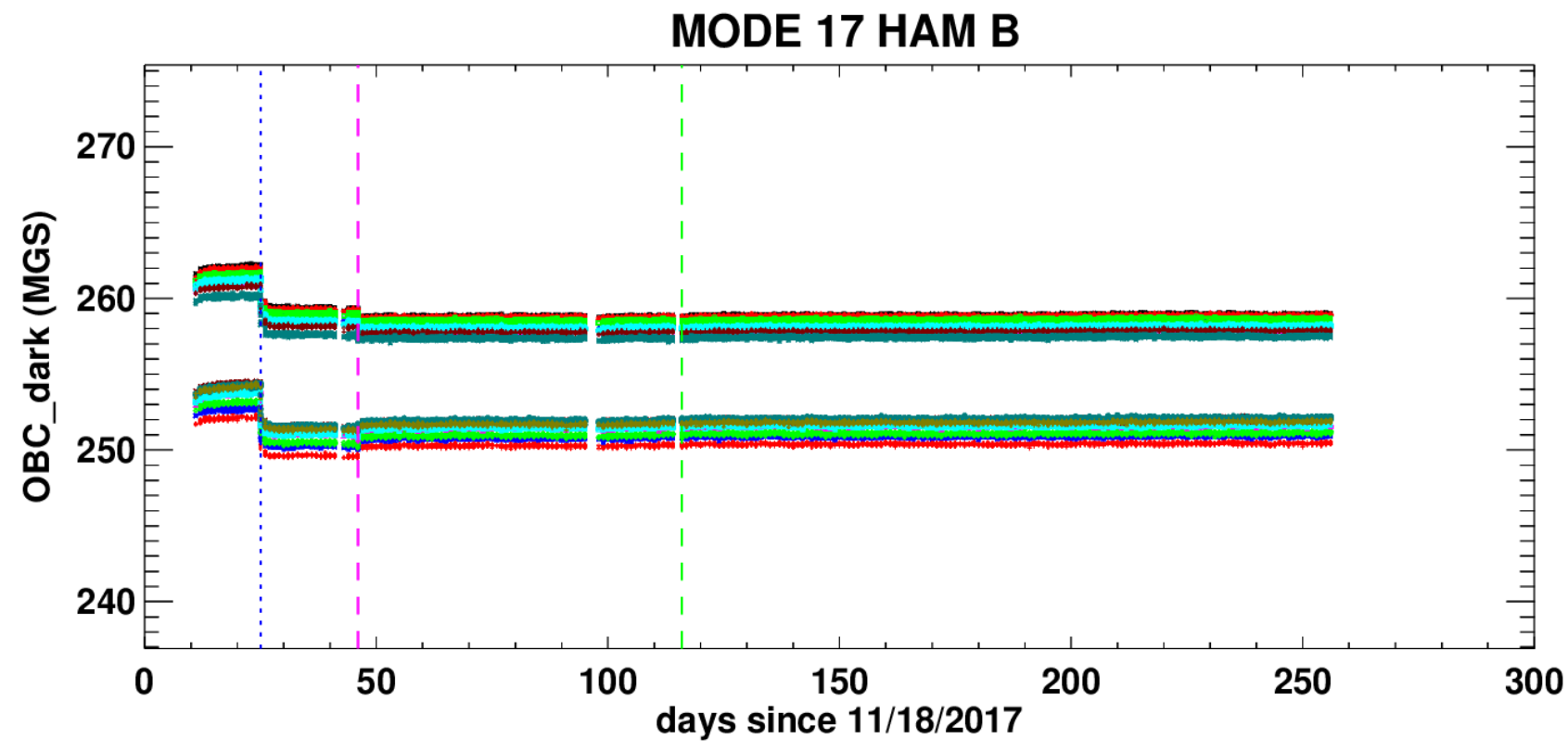

Figure 10 DNB dark offset trending in mode-17 and ham side-B 
MODE 32 HAM A

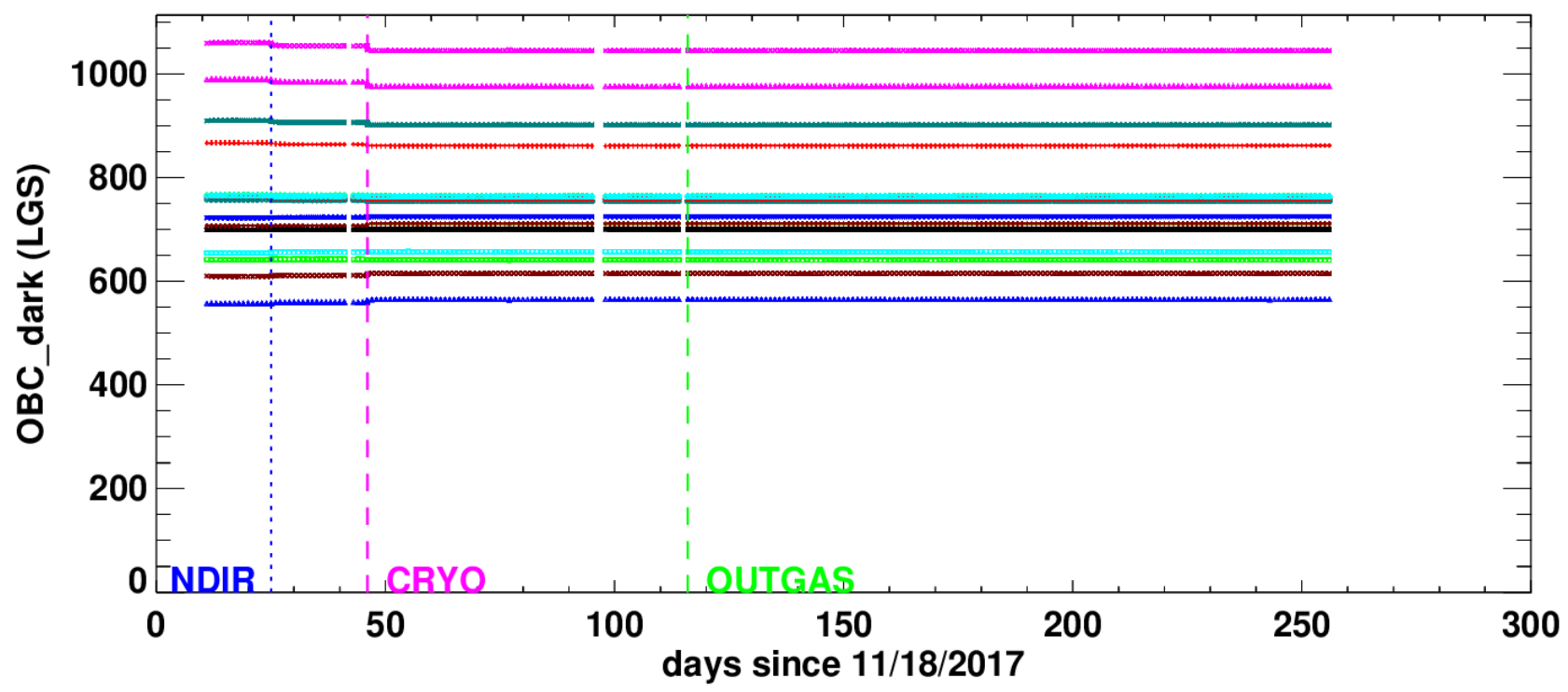

Figure 11 DNB dark offset trending in mode-32 and ham side-A.

Similar to the S-NPP, JPSS-1 DNB has design requirements on signal-to-noise ratio (SNR) at the minimal radiance, which happens in HGS, specifically, the minimal radiance is referred as the quarter moon radiance, roughly about $3 \times 10^{-9}$ $\mathrm{W} \cdot \mathrm{cm}^{-2} \mathrm{sr}^{-1}$. Figure 12 shows the averaged SNR in modes $1-21$, where the specification is $\mathrm{SNR}=6$. Obviously, all aggregation modes satisfy the system design specifications in this chart.

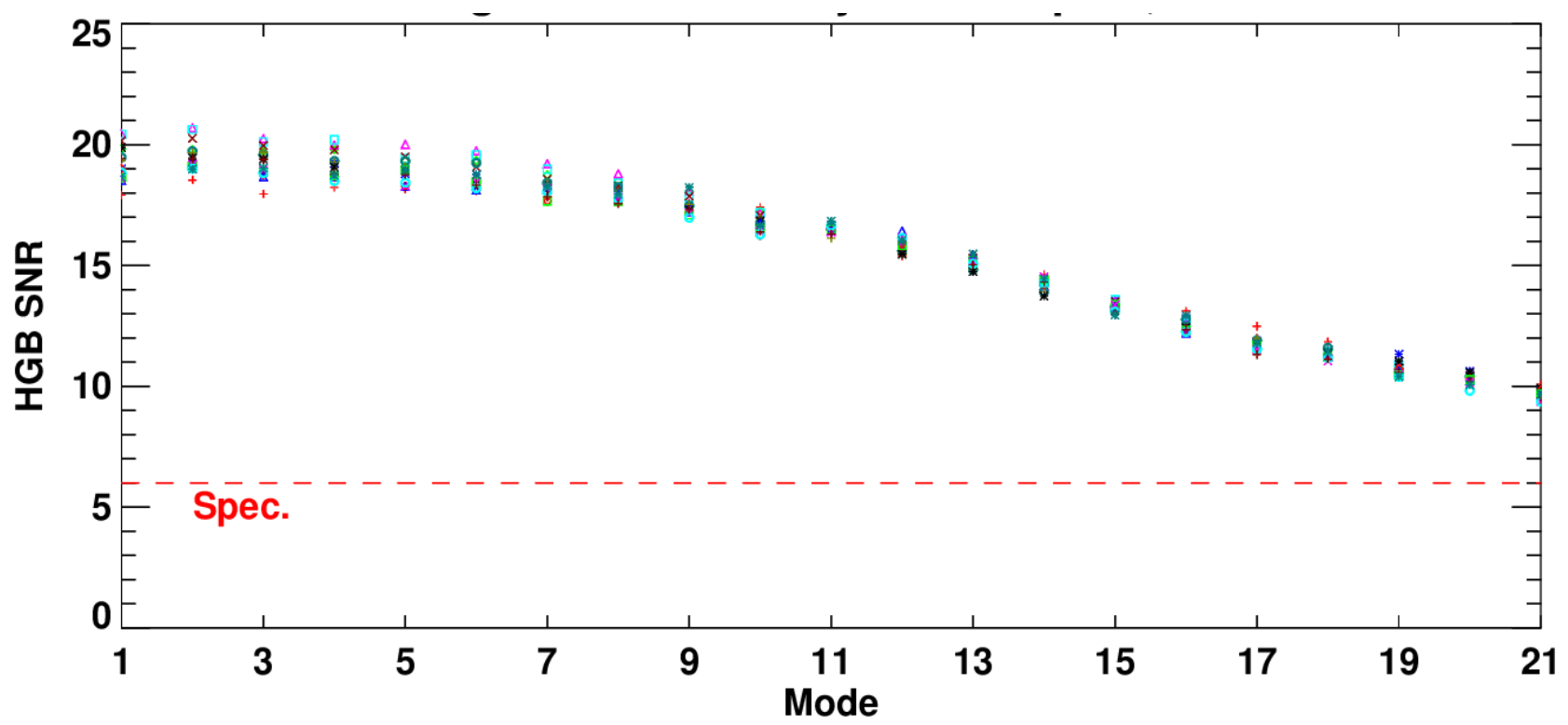

Figure 12 DNB HGS SNR at minimal radiance. 


\section{COMPARISON STUDIES}

The currently performed calibration method is conducted by the IDPS, where two VROPs of determining the gain ratios between the different DNB gain stages (VROP705), and dark offsets (VROP702). The drawback is mentioned that the nighttime airglow will contaminate the VROP data so as to introduce additional uncertainty in EV data. In this section, we will present the calibration comparison results.
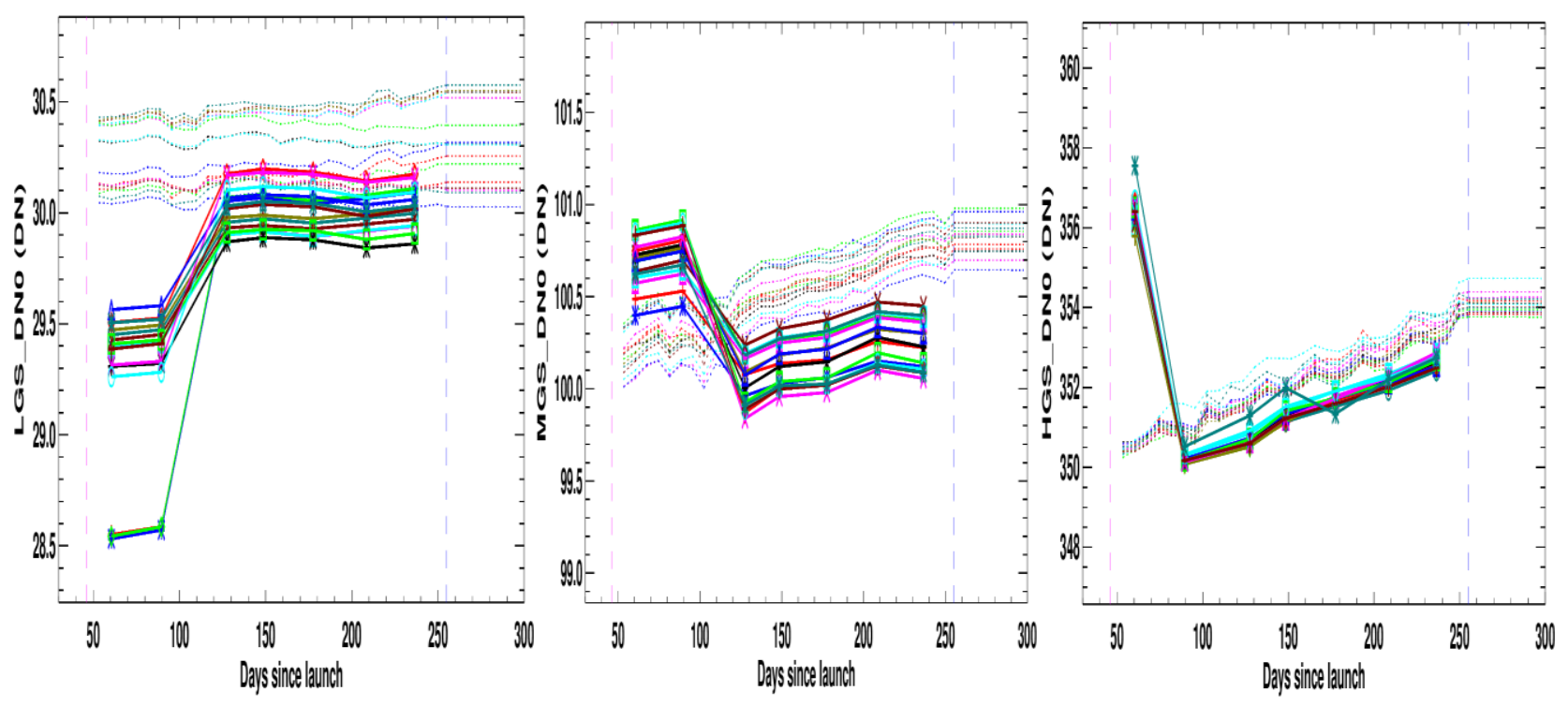

Figure 13 DNB dark-offset comparisons to IDPS: all detectors in mode-1 for LGS, MGS and HGS. Solid lines are from IDPS, while dotted lines are from VCST.

Figure 13 shows the VCST estimated DNB dark-offset comparisons to IDPS. The solid lines in these charts are the IDPS LUTs and dashed lines are VCST LUTs. There are seven dark-offset LUTs provided by IDPS since the cryo-door open. Note that the first two IDPS LUTs show large differences, after that, our results and IDPS LUTs show similar trending, and the relative difference is less than $0.5 \%$ for HGS and MGS, and $1.0 \%$ for LGS. The similar observations can be noticed in other modes as well. For example, Figure 14 show the case of mode-32, the highest node to deal with the edge pixels. Both Figure 13, and Figure 14 illustrate the facts of the first two dark-offset IDPS LUTs are not accurate with large inconsistent biases. 

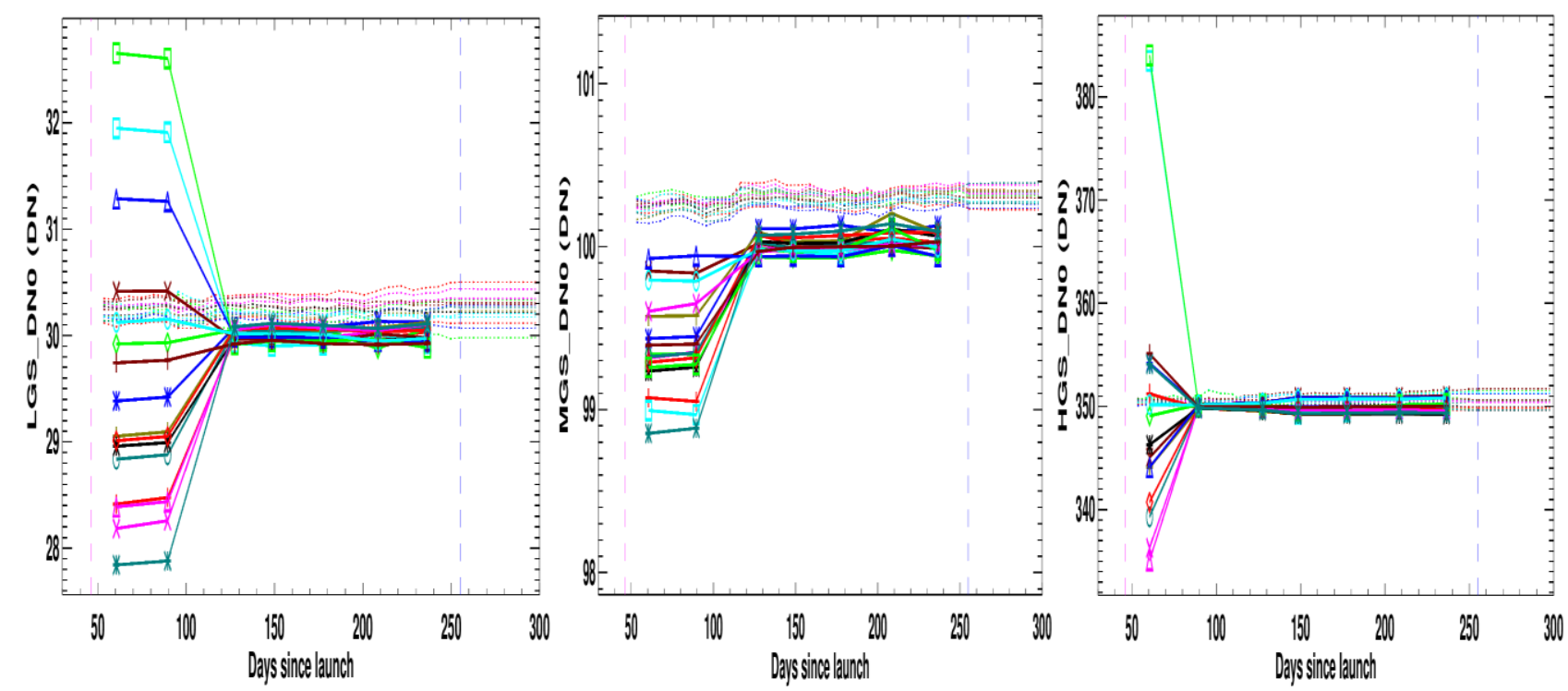

Figure 14 DNB dark-offset comparisons to IDPS: all detectors in mode-32 for LGS, MGS and HGS. Solid lines are from IDPS, while dotted lines are from VCST.

So far, there are only two IDPS LGS gain LUTs available since the year 2018. Figure 15 shows the comparison results in mode-1, mode-16, and mode-32 from the left to the right. There are large difference for the first LGS gain LUT between IDPS and VCST results. However, we got very good matches at the second LGS gain LUT, such that the relative difference is less than $0.2 \%$ for all detectors, modes and ham sides. Note that the LGS gain trend is stable for all modes and detectors. Again, the vertical line denotes the most recent measurement day, and a linear fit is used to extend for the forward delivery.
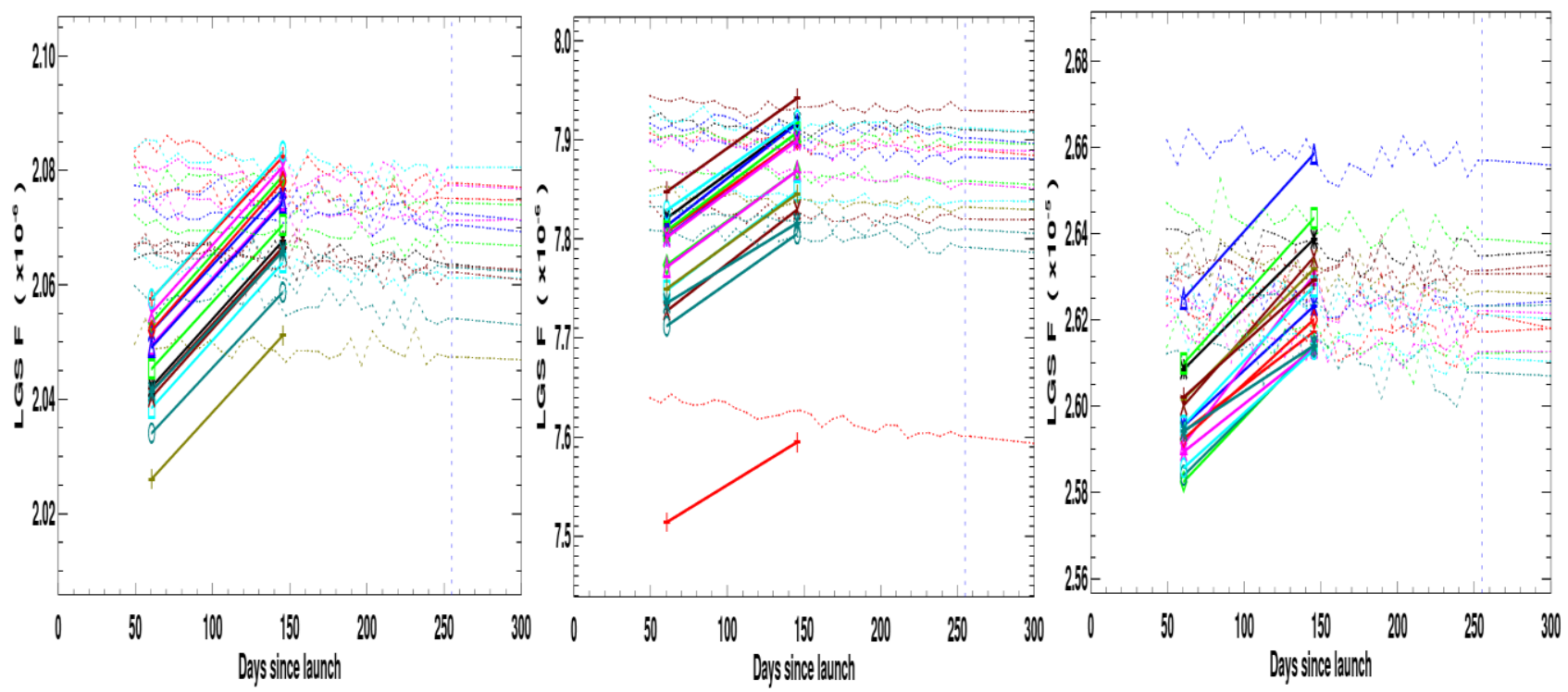

Figure 15 DNB dark-offset comparisons to IDPS: all detectors of mode-32 in LGS, MGS and HGS. Solid lines are from IDPS, while dotted lines are from VCST. 


\section{CONCLUDING REMARKS}

In the early mission, the JPSS-1 VIIRS DNB calibration has been successfully performed. This ultra-sensitive DNB calibration is conducted for all aggregation modes, detectors, gain stages and HAM-sides. In this paper, recent DNB onorbit performance has been presented. The overall on-orbit performance of DNB shows that degradation behavior is normal. Both DNB LGS gain and dark offset have been in very stable trending. DNB HGS SNR at minimal radiance satisfy the system design specifications. Agreement with the recent IDPS results is shown, such that DNB LGS gains are under $0.2 \%$ difference for all modes, ham sides and detectors. DNB dark offsets are matched well.

\section{ACKNOWLEDGEMENTS}

The authors would like to thank other members of the VCST for their technical discussions and assistance, especially Dr. Kevin Twedt for his helpful comments.

\section{REFERENCES}

[1] H. Chen, X.Xiong, C. Sun, X. Chen and K. Chiang, "Suomi-NPP VIIRS day-night band on-orbit calibration and performance", Journal of applied remote sensing, Vol. 11(3), 036019 (2017).

[2] J. Geis, C. Florio, D. Moyer, K. Rausch and F. Luccia, "VIIRS Day-Night Band Gain and Offset Determination and Performance", Proc. Of SPIE, vol.8510, 851012, pp.1-12, 2012.

[3] S. Lee, K. Chiang, X. Xiong, C. Sun and S. Anderson, "The S-NPP VIIRS Day-Night Band On-Orbit Calibration/Characterization and Current State of SDR Products", Remote Sensing, 2014, v6, pp12427-12446, ISSN 2072-4292.

[4] K. Rausch, S. Houchin, J. Cardema, G. Moy, E. Haas and F. Luccia, "Automated Calibration of the Soumi National Polar-orbiting Partnership (S-NPP) Visible Infrared Imaging Radiometer Suite (VIIRS) Reflective Solar Bands", Journal of Geophysical Research: Atmospheres, v118, pp.13434-13442, 2013.

[5] N. Lei, B. Buenther, Z. Wang and X. Xiong, "Modeling SNPP VIIRS Reflective Solar Bands Optical Throughput Degradation and Its Impacts on the Relative Spectral Response", Proc. SPIE, vol.8866, paper 55, 2013.

[6] S. Millers, S. Weiss and K. Liang, "VIIRS day/night band (DNB) stray light characterization and correction", Proc. SPIE 2013, doi:10.1117/12.2023107.

[7] C. Cao and Y. Bai, "Quantitative Analysis of VIIRS DNB Nightlight Point Source for Light Power Estimation and Stability Monitoring", Remote Sensing, 2014, v6, pp.11915-11935.

[8] S. Lee, and C. Cao, "Soumi NPP VIIRS Day/Night Band Stray Light Characterization and Correction Using Calibration View Data", Remote Sensing, 2016, v8, pp138; doi: 10.3390/rs8020138.

[9] L.B. Liao, S. Weiss, S. Mills and B. Hauss, "Soumi NPP CIIRS Day-Night Band On-orbit Performance", Journal of Geophysical Research: Atmospheres, v118, pp.12705-12718, 2013.

[10] S. Lee, C. Sun, K Chiang and X. Xiong, "An Overview of NASA VIIRS Day-Night Band (DNB) On-orbit Radiometric Calibration”, Proc. SPIE 2014, 9218, doi:10.1117/12.2061912. 